\title{
Bacopa monnieri supplementation increases learning and short-term memory retention of sleep-deprived Drosophila melanogaster
}

\author{
Bradley Ashley Gue Ong ${ }^{1}$, Maria Clarice Nuqui Villanueva ${ }^{2}$, Paul Mark Baco Medina ${ }^{1^{*}}$ \\ ${ }^{1}$ Biological Models Laboratory, Department of Biochemistry and Molecular Biology, College of Medicine, University of the Philippines Manila, Manila City \\ 1000, Philippines. \\ ${ }^{2}$ College of Medicine, Pamantasan ng Lungsod ng Maynila, Manila, Philippines.
}

\begin{tabular}{l}
\hline ARTICLE INFO \\
\hline Received on: $14 / 06 / 2020$ \\
Accepted on: $22 / 10 / 2020$ \\
Available online: $05 / 12 / 2020$
\end{tabular}

Key words:

Aversive phototaxic

suppression, Drosophila

melanogaster, Bacopa

monnieri, short-term memory.

\begin{abstract}
Aversive phototaxic suppression (APS) assay is a behavioral assay in which photopositive fruit flies (Drosophila melanogaster) are trained to avoid light using quinine as an aversive stimulus. This conditioned behavior is found to be impaired by sleep deprivation in D. melanogaster. This study tested whether supplementation with Bacopa monnieri can counter this impairment. Three sublethal and effective concentrations of B. monnieri supplement $\left(4.62 \times 10^{-4} \mathrm{~g} /\right.$ $\mathrm{ml}, 4.62 \times 10^{-3} \mathrm{~g} / \mathrm{ml}$, and $4.62 \times 10^{-2} \mathrm{~g} / \mathrm{ml}$ ) were given to test groups of non-sleep-deprived (slp + ) and sleep-deprived $\left(\mathrm{slp}^{-}\right)$flies subjected to the APS assay. The extent of learning and short-term memory (STM) retention was based on average pass rates (APRs) defined as the number of trials that each fly exhibited a photonegative response before and after conditioning. B. monnieri supplement improved the APR of both slp + and slp- flies as compared to the unsupplemented flies. These effects were found to be dose-dependent from the low- to the mid-level $\left(4.62 \times 10^{-4} \mathrm{~g} / \mathrm{ml}\right.$ to $4.62 \times 10^{-3} \mathrm{~g} / \mathrm{ml}$ ) concentrations of the supplement on D. melanogaster learning and STM retention. Supplementing fly food with $B$. monnieri improved learning and STM of slp- flies with learning and STM deficits caused by sleep deprivation. The slp- flies given $B$. monnieri supplementation had higher learning rates and longer STM retention than slp+ flies without B. monnieri supplementation. Unexpectedly, slp- flies given B. monnieri supplementation had the same learning and STM retention levels as slp+ flies also given B. monnieri supplementation. Bacopa monnieri supplementation enhances both the learning capacities and the STM retention of both non-sleep-deprived and sleepdeprived fruit flies. Advantageous effects were dose-dependent for both learning and STM retention.
\end{abstract}

\section{INTRODUCTION}

Sleep deprivation leads to significant changes in hippocampal activity during episodic memory encoding, which results in impaired subsequent retention (Havekes and Abel, 2017). Situations like shift works and jet lags are associated with sleep deprivation and fatigue (Thomas et al., 2020), which leads to decrements in cognition as well as in psychomotor vigilance response speed or the reaction time to sustained attention to visual stimulus (Belenky et al., 2003; Gregory et al., 2015; Waser et al., 2019). Nonprescription supplements, such

${ }^{*}$ Corresponding Author

Paul Mark Baco Medina, Biological Models Laboratory, Department of Biochemistry and Molecular Biology, College of Medicine, University of the Philippines Manila, Manila City 1000, Philippines.

E-mail:pmbmedina@post.upm.edu.ph as nootropics, have been increasingly commonplace in these situations to combat the effects of sleep deprivation (Klumpers et al., 2015). Nootropics, also known as memory-enhancing drugs, are purported to boost levels of neurotransmitters in the brain for better learning and memory retention (Brondino et al., 2013; Yang et al., 2020). However, the link between these compounds and benefits to cognition still remains unestablished (Medina et al., 2015).

The plant Bacopa monnieri is a commonly used natural nootropic in Ayurvedic medicine. Bacopa monnieri is also used either alone or in combination with other herbs, as a memory and learning enhancer, sedative, and antiepileptic (Bhattacharya et al., 2000; Dubey and Chinnathambi, 2019). Studies of B. monnieri whole plant or alcohol extracts used in rats have reported cognitionenhancing effects, including improved motor learning and acquisition, consolidation, and retention of memory (Abdul Manap et al., 2019; Bhattacharya and Ghosal, 1998; Chaudhari et al., 2017). 
The use of Drosophila melanogaster as an organism for the study of learning and memory has been established extensively. They can be reliably trained to learn and remember the association between stimuli through both classical and operant conditioning (Foley et al., 2017; Dissel, 2020; McGuire, 2005). Many of the molecular mechanisms underlying learning and memory in mammalian systems were first elucidated on the fly (Dag, 2019; Dissel, 2020; Margulies, 2005). The pattern of learning and memory functions is conserved between mammals and Drosophila (Guarnieri and Heberlein, 2003; Tsuda and Lim, 2018). The fly has short- and long-term memory involving acquisition, consolidation, and recall. Importantly, its cognitive capacities are shown to be highly amenable to experimental alterations on the genetic, biochemical, environmental, and pharmacological levels.

The behavioral performance of $D$. melanogaster individuals in the aversive phototaxic suppression (APS) assay provided information on the effects of $B$. monnieri supplementation on non-sleep-deprived $\left(\operatorname{slp}^{+}\right)$and sleep-deprived (slp-) Drosophila. Future efforts on the animal model may relate the results obtained to better evaluate the effects of these nootropics among humans in conditions like jet lag, sleep deprivation, and night shift-requiring jobs.

This study tested the potential effects of B. monnieri supplement on the learning capacities and short-term memory (STM) retention in non-sleep-deprived and sleep-deprived $D$. melanogaster subjected to the APS assay.

\section{MATERIALS AND METHODS}

\section{Fruit fly breeding and handling}

Wild-type Drosophila melanogaster (Oregon-R-P2 strain) were obtained from the Bloomington Drosophila Stock Center (Indiana, USA). The flies were reared based on the protocol by Medina et al. (2015) at room temperature on a sweet potato medium. A bottle containing 1-day-old flies from hatched pupae was immediately transferred to two separate bottles containing freshly prepared sweet potato medium. The flies were maintained at $24^{\circ} \mathrm{C}-28^{\circ} \mathrm{C}$ and in a 12 -hours light/12-hours dark cycle, with the $40 \mathrm{~W}$ fluorescent light. Only female flies were used in the experiments since they exhibit sustained activity during the day, whereas male flies spend considerable time sleeping during both day and night (Huber et al., 2004). Thus, female flies are more consistent models for sleep-related studies (Dissel, 2020; Li et al., 2009).

Carbon dioxide gas was used to anesthetize the flies during the segregation of males and females based on the presence or absence of sex combs. Newly enclosed flies were separated from breeding bottles and transferred and kept in vials with media for 7 days to ascertain that all flies that were used in the experiment were 7 days old.

\section{Determination of B. monnieri experimental doses}

A lethality test was carried out to determine the experimental doses of B. monnieri supplement (Memo Plus Gold, Puducherry, India). The baseline dosages of the supplements for Drosophila were calculated based on a study by Nair and Jacob (2016) for dose conversion between humans and animals. We focused our doses based on a rough extrapolation from the prescribed human dosing. Additionally, we used flanking doses both 10 times greater and 10 times less than our estimated human dose. An assumption for extrapolation was that a $70 \mathrm{~kg}$ human equals a $1 \mathrm{mg}$ fly. To evaluate the effect of $B$. monnieri supplementation, a $4.62 \mathrm{~g} / \mathrm{ml}$ value was computed relative to the weight of the flies, equivalent to $125 \mathrm{mg}$ for human (the dose is administered to humans twice a day). Two tenfold higher values and two tenfold lower values were included in the series of test dosages for the lethality test. Three sublethal concentrations were chosen for the experiment proper: $4.62 \times 10^{-4} \mathrm{~g} / \mathrm{ml}, 4.62 \times 10^{-3} \mathrm{~g} /$ $\mathrm{ml}$, and $4.62 \times 10^{-2} \mathrm{~g} / \mathrm{ml}$, and were labeled as low, mid, and high, respectively.

Flies were raised in the media containing a specific dose of the $B$. monnieri supplement at $25^{\circ} \mathrm{C}$. The supplement was added into the media when it cooled down to $60^{\circ} \mathrm{C}$ and before it solidified.

\section{Sleep deprivation procedure}

Prior to sleep deprivation, 7-day-old female flies were individually selected for positive phototaxis. The flies, fed with and without $B$. monnieri supplement, were sleep-deprived using a sleep nullifying apparatus (SNAP) adapted from Shaw et al. (2000). The flies were individually placed inside test tubes that contain appropriate media and were positioned on the rack of the SNAP. The SNAP moves the rack up and down every 4 seconds such that flies in the test tubes were displaced during the dropping movement and prevented from sleeping. The sleep deprivation process was done for 10 hours (12:00 AM to 10:00 AM).

\section{Sensitivity tests for confounding stress}

\section{Quinine sensitivity}

A $1 \times 4-\mathrm{cm}$ filter paper was soaked in $1 \mu \mathrm{M}$ quinine solution (Sigma-Aldrich, St. Louis, MO) and placed inside the lighted tube of the T-maze, two centimeters from the opening of the tube. The other tube was covered in aluminum foil to serve as the darkened tube. These two tubes were screwed on opposite sides of the center column of the T-maze.

A fly was placed inside the dark tube for 5 minutes before being allowed to move to the lighted tube. The time in seconds it took for the fly to move toward the lighted tube with quinine was recorded. This time is the quinine sensitivity index (QSI). The QSI was determined by calculating the time in seconds that the fly spent on the dry side of the tube when the other side had been wetted with quinine, during a 5-minutes period.

\section{Velocity stress}

Seven-day old female flies were continuously displaced using the SNAP, 30 times per minute for 10 hours from 12:00 AM to 10:00 AM. After the SNAP treatment, the flies were then given 30 minutes to recover before being subjected to the APS assay.

\section{APS assay}

Learning phase

A fly that demonstrated positive phototaxis was tapped back into the dark chamber. Thirty seconds was allowed for acclimatization but during this time, a filter paper with a quinine solution was put inside the lighted chamber. Then, the trap door 
was slowly opened as well as the lights. The fly was given 10 seconds to walk toward the quinine coated filter paper in the lighted tube. This was repeated nine times. Failure to walk to the lighted tube was recorded as "Pass," which is equivalent to "task learned through reinforcement." The right-left double alternation of the tubes was executed in an RRLLRRLLRR manner, switching every two trials.

\section{STM retention phase}

To assess STM retention in flies, each fly was placed back into its original food tube and remained there for 3 hours. Nootropic feeding was resumed during this resting period. Three hours after training, each fly was put in the T-maze again in the same way as before, and the number of times the fly avoids (pass) or goes into (fail) the lighted chamber was recorded. A total of five trials were conducted to test STM retention. Alternation of the tubes was carried out after every trial.

\section{Average pass rates}

Flies from the darkened tube which went through the lighted tube were recorded and tallied per trial except during photopositive screening. A score of "1" was given for a fly exhibiting a photonegative choice. A score of " 0 " was noted for a fly entering the lighted tube. From a series of nine trials, any consecutive trials that obtained a score of " 1 " at least four consecutive times, starting from the last trial (ninth trial), were summed and divided by the total number of trials to get the pass rate. The APR or the mean of all the pass rates of the 30 flies per treatment group was then obtained together with its standard deviation and error. These values were then used to represent the percent learning and memory retention in the descriptive statistical methods that were carried out.

\section{Statistical analysis}

A two-way analysis of variance (ANOVA) was utilized to detect if the differences in obtained APRs were significant due to $B$. monnieri supplementation, sleep deprivation, and the interaction of the two. In addition, the Bonferroni method was used as a pairwise comparison for each treatment group using the level of significance $(\alpha)$ which was set at 0.05 for all statistical tests used.

\section{RESULTS AND DISCUSSION}

This study presents the first report, to our knowledge, on the effects of $B$. monnieri supplementation in learning and in STM of sleep-deprived flies. In this study, we utilized Drosophila as a model of learning and memory impairment caused by sleep deprivation and hypothesized that $B$. monnieri may counter the impairment brought by sleep deprivation. Our study showed that sleep deprivation reduced both learning and STM retention in flies. With B. monnieri treatment, results showed that B. monnieri supplement improved the learning and the STM retention of both sleep-deprived and non-sleep-deprived flies. Future efforts on the animal model may then relate the results obtained to better evaluate the effects of these nootropics on humans in conditions like jet lag, sleep deprivation, and night shift-requiring jobs.

\section{Bacopa monnieri supplementation converted to fly dosages is sublethal}

The lethality test showed that the negative control (i.e., no B. monnieri supplement) had a $100 \%$ survival rate after seven days (Figure 1). The three lower concentrations, 4.62 $\times 10^{-4} \mathrm{~g} / \mathrm{ml}, 4.62 \times 10^{-3} \mathrm{~g} / \mathrm{ml}$, and $4.62 \times 10^{-2} \mathrm{~g} / \mathrm{ml}$, among the five concentrations tested exhibited survival rates close to the negative control, with the highest of these three concentrations obtaining $86.67 \%$ survival rate or 26 out of 30 flies. These three concentrations were determined to be sublethal and used further in the next phase of the experimentation. The remaining two higher concentrations, $4.62 \times 10^{-1} \mathrm{~g} / \mathrm{ml}$ and $4.62 \mathrm{~g} / \mathrm{ml}$, of the five concentrations tested, had survival rates of $60.00 \%$ and $53.33 \%$, respectively. In addition, the pupae formation from these two higher concentrations was approximately 50\% less compared to the three lower concentrations, suggesting that these two concentrations may have negative effects on fertility and thus they were not used further in succeeding experiments.

Although the use of $B$. monnieri has been reported to exert different pharmacological actions, there is evidence that some can cause toxicity (Singh and Dhawan, 1982; Singh and Singh, 2009). The lethality test is intended to give information on the safety dosage of $B$. monnieri before further evaluation of its benefits in fruit flies. Several studies have demonstrated the safety profile of B. monnieri in mammals (Rauf et al., 2012; Sireeratawong et al., 2016), similar to the sublethal results in our study. The decrease in pupae formation is similar to the studies on mammals (Singh and Singh, 2009). Constant high dose administration of B. monnieri demonstrated a decrease in sperm motility, viability, and sperm count. It also showed minute histological changes intraepithelial vacuolation, loosening of germinal epithelium, exfoliation of germ cells, and occasionally giant cell formation. However, it is noteworthy that libido remained to be unaffected in B. monnieritreated rats.

\section{Quinine sensitivity and velocity stress does not affect learning capacity and memory formation}

Quinine sensitivity was tested in order to assess its induction of avoidance behavior in both slp + and slp- flies. It was observed that when quinine was used in the T-maze test, flies briefly crossed from the dark chamber of the tube to the quinine portion of the lighted chamber and then quickly came back to the dry side of the chamber. There was no significant difference $(p<$ 0.05) on the QSI between slp+ and slp- fruit flies (Table 1). All flies in both treatment groups displayed QSI scores well within ranges that permit normal learning together.

Table 1. Difference on the QSI of both undeprived and sleep-deprived $D$. melanogaster.

\begin{tabular}{lc}
\hline Fly type $(\boldsymbol{n}=\mathbf{3 0})$ & QSI \\
\hline Non-sleep-deprived D. melanogaster & $4: 29 \pm 0: 13$ \\
Sleep-deprived D. melanogaster & $4.34 \pm 0: 18$ \\
\hline
\end{tabular}

All flies display QSI scores well within ranges that permit normal learning. The final QSI is the average of the scores obtained for 30 flies \pm standard deviation (SD) values. 
Since there is no difference in quinine sensitivity of slp+ and slp- flies, these data suggest that contact chemosensation of the bitter-tasting compound is not significantly altered by sleep deprivation.

Studies have revealed that varying intensity of stress can both improve and impair learning and memory (Duncko et al., 2007; Fukui et al., 2001). When the velocity stress test was carried out, the APR of the fruit flies that had undergone the stress test was similar to the APR values of the $\mathrm{slp}^{-} / \mathrm{Bm}^{-}$treatment group. ANOVA results indicated that the increased rotations per minute of the sleep deprivation machine had no significant difference $(p<0.05)$ on the learning and STM retention capacity of the fruit flies (Fig. 2). This indicates that the reduction of learning

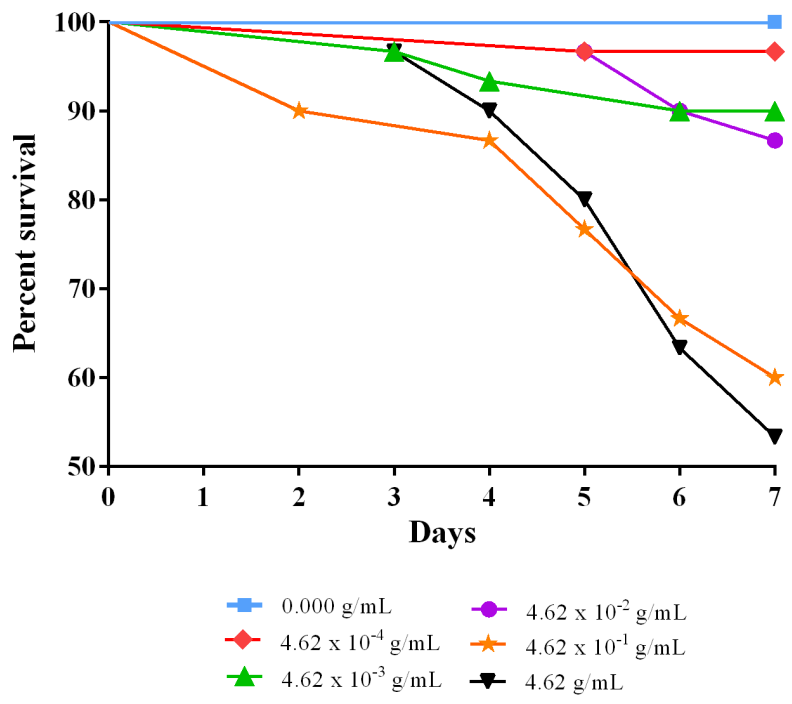

Figure 1. Percent survival of Drosophila melanogaster on the difference of tenfold concentration of Bacopa monnieri supplementation over the course of 7 days. The three lowest among the five tested concentrations showed the highest survival rate next to the no supplement treatment group.

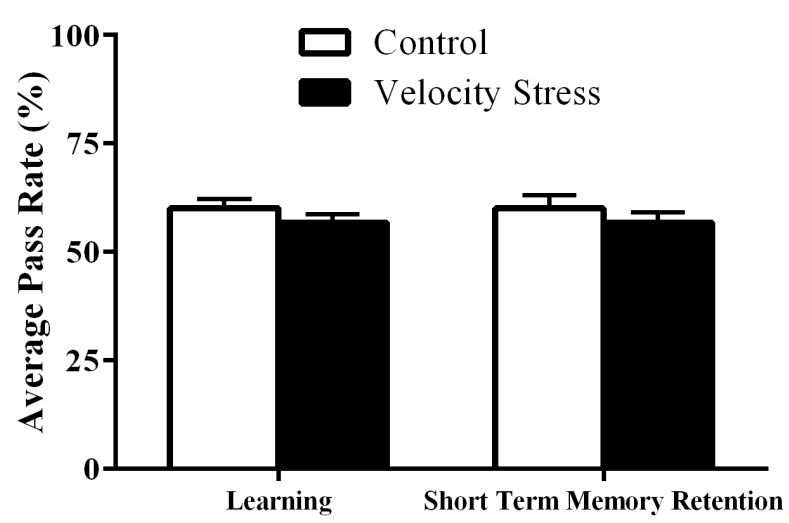

Figure 2. Comparison of the effect of velocity stress caused by mechanical stimulation of the machine on the learning and STM retention of D. melanogaster $(n=30)$. The results indicate that stress did not affect both learning and memory $(p<0.05)$ capacity and STM retention is specifically due to sleep deprivation alone. Despite velocity stress having the same intensity as sleep deprivation in flies (Shaw et al., 2000), our results showed that learning and STM capacity of flies that had undergone the test remained intact compared to the $\mathrm{slp}^{-} / \mathrm{Bm}^{-}$treatment group. Flies are known to sleep mostly at night (Huber et al., 2004); hence, learning and STM impairment are likely to be secondary from sleep deprivation rather than from mechanical stress.

\section{Sleep deprivation reduces learning capacity and STM retention}

Reduction of memory retention has been previously documented in flies (Dag, 2019; Bai and Sehgal, 2015). However, these studies did not account for learning alongside memory retention. The APS assay was used to assess learning capacity along with STM in the context of sleep deprivation. APR values of all sleep-deprived treatment groups for both learning and STM retention significantly decreased $(p<0.05)$ in comparison with the APR values of non-sleep-deprived groups. The APR values of the $\mathrm{slp}^{-} / \mathrm{Bm}^{-}$treatment group for learning and STM retention were $45.92 \%$ and $47.33 \%$, respectively (Fig. 3 ).

The number of trials it takes for a specific fly to learn in the $\mathrm{slp}^{-} / \mathrm{Bm}^{-}$treatment group was significantly lower $(p<$ 0.05 ) from the $\mathrm{slp}^{+} / \mathrm{Bm}^{-}$group. This finding indicates that low motivation is an unlikely reason for the impairment of learning. On the other hand, the decline in STM retention may be attributed to the deficit in mushroom body (MB) plasticity (Seugnet et al., 2009) during episodic memory encoding, resulting in impairment of subsequent retention. Two factors are most likely to contribute to learning and memory deficits following sleep loss: excessive synaptic potentiation and dysregulated reinforcement from dopaminergic neurons in the MB. In Drosophila, dopaminergic neurons project arborizations to the MB neuropile where they influence aversive learning (Seugnet et al., 2009). Previous studies have shown that dopaminergic neurons in the MBs play a role in decision-making under conflicting situations (Tang and Guo, 2001) and may signal the aversive stimulus to the MBs in

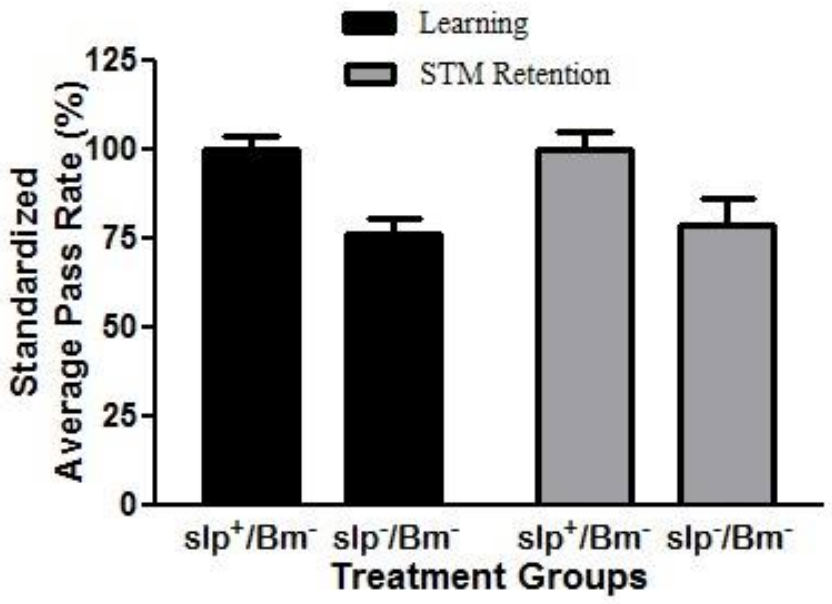

Figure 3. APR values for learning and STM retention of sleep-deprived $D$. melanogaster show impaired learning and STM in Drosophila. There is a decrease $(p<0.05)$ in learning and STM retention in sleep-deprived flies compared to non-sleep-deprived flies $(n=30)$. 
olfactory conditioning (Riemensperger et al., 2005). Interestingly, flies in the APS assay also face a conflicting choice between their prepotent attraction toward light and the aversive stimulus. The decrease in learning and STM retention in sleep-deprived fruit flies is similar to cognitive impairments observed in sleepdeprived humans (Belenky et al., 2003).

Supplementation of B. monnieri increases learning in nonsleep-deprived and sleep-deprived flies

The B. monnieri supplement is purported to enhance learning. All treatment groups with $B$. monnieri supplementation had increased APR values in comparison to the one without $B$. monnieri supplementation. The $\operatorname{slp}^{+} / \mathrm{Bm}^{+}$mid concentrationtreatment group obtained the highest APR of $81.48 \%$ (Fig. 4). The mid and high concentration APR values were significantly higher $(p<0.05)$ than the APR values of the low concentration. However, the difference between the mid and high concentrations was found to be not significant $(p>0.05)$. Although the APR values obtained in the $\mathrm{slp}^{-} / \mathrm{Bm}^{+}$groups were lower compared to the APR values of the $\operatorname{slp}^{+} / \mathrm{Bm}^{+}$groups (Fig. 5), this difference was not significant ( $p$ $>0.05)$. There was no interaction between sleep deprivation and $B$. monnieri supplementation that may affect the rates obtained ( $p$ $<0.01)$.

The B. monnieri supplement increases learning in sleep-deprived flies at the same level as their non-sleep-deprived counterparts even at low concentration. The results demonstrate that B. monnieri supplement makes up for the deficiency of learning caused by sleep deprivation. The increase in learning of flies fed with B. monnieri was dose-dependent, where the optimal effect was reached at the mid concentration used. The groups fed with $B$. monnieri supplement had a faster learning rate in the learning phase (phase I), where evidence of learning was observed

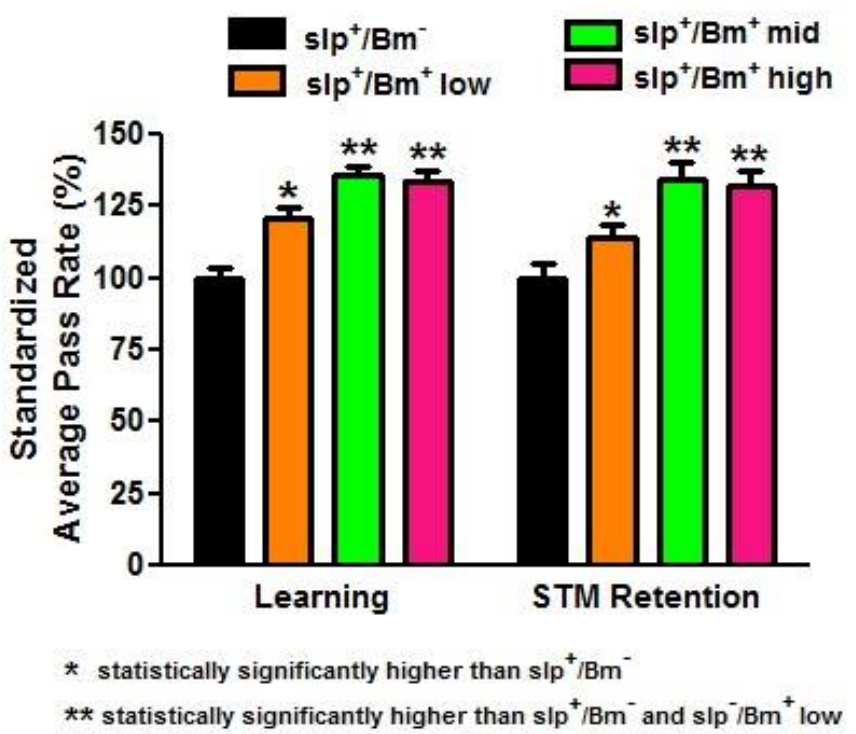

Figure 4. APR values for learning and STM retention of non-sleep-deprived $D$. melanogaster show an increase $(p<0.05)$ in learning and STM retention in $\mathrm{slp}^{+}$ $\mathrm{Bm}^{+}$compared to $\operatorname{slp}^{+} / \mathrm{Bm}^{-}(n=30)$. Low dosage corresponds to $4.62 \times 10^{-4} \mathrm{~g} /$ $\mathrm{ml}$, middle dosage corresponds to $4.62 \times 10^{-3} \mathrm{~g} / \mathrm{ml}$ and high dosage corresponds to $4.62 \times 10^{-2} \mathrm{~g} / \mathrm{ml}$. Error bars represent standard error (SE) values. as early as trial 2 . This is indicative of better learning capacities of flies supplemented with $B$. monnieri than flies without $B$. monnieri supplement.

Phytochemical studies have revealed different secondary metabolites in B. monnieri, like alkaloids, saponins, and sterols (Deepak and Amit, 2004; Mahato et al., 2000). Previous studies of $B$. monnieri have identified its effects on learning acquisition. Learning ability in rats has been significantly enhanced by Bacopa extract as it facilitated acquisition, consolidation, and retention of three newly learned behavioral responses (Singh and Dhawan, 1982). Additionally, this study showed that the bacosides caused enhanced levels of protein kinase activity and increased protein levels in the hippocampus, cerebral cortex, and hypothalamus regions of the brain. The findings indicated positive implications for improved neurotransmission and repair of damaged neurons via enhanced regeneration of nerve synapses. A study by Hosamani and Muralidhara (2009) elucidated that B. monnieri prevented apoptosis in neurons from oxidative stress, a process that may be induced by sleep deprivation in learning of fruit flies.

Supplementation of B. monnieri increases STM in non-sleepdeprived flies and sleep-deprived flies

The determination of STM retention in slp + and slpflies fed with B. monnieri also used the APS assay similar to the learning phase done earlier. In this assay, a period of 3-hours posttraining was allowed to pass before starting the memory retention phase. There was a significant increase $(p<0.001)$ in

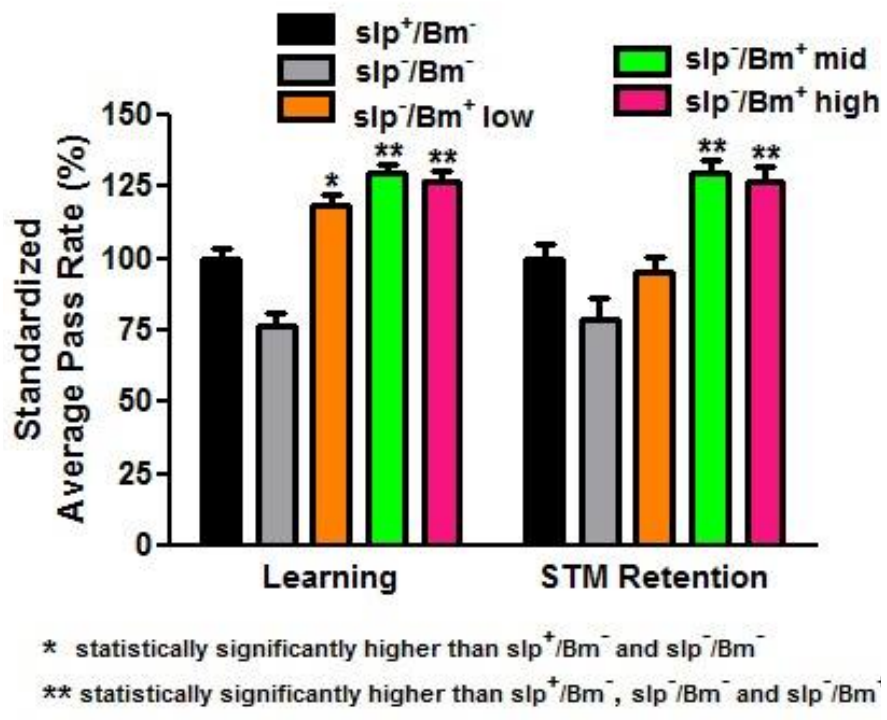

Figure 5. A B. monnieri altered learning and memory of D. melanogaster APR values of all sleep-deprived treatment groups decreased in comparison to the APR values of non-sleep-deprived groups. All treatment groups with $B$. monnieri supplementation had increased $(p<0.05)$ APR values in comparison to the no supplementation group $(n=30)$. Low dosage corresponds to $4.62 \times 10^{-4} \mathrm{~g} /$ $\mathrm{ml}$, middle dosage corresponds to $4.62 \times 10^{-3} \mathrm{~g} / \mathrm{ml}$, and high dosage corresponds to $4.62 \times 10^{-2} \mathrm{~g} / \mathrm{ml}$. Error bars represent SE values. 
the APR values of the groups $\operatorname{slp}^{+} / \mathrm{Bm}^{+}$low, $\operatorname{slp}^{+} / \mathrm{Bm}^{+}$mid, and $\mathrm{slp}^{+} / \mathrm{Bm}^{+}$high in comparison to $\operatorname{slp}^{+} / \mathrm{Bm}^{-}$(Fig. 4). This implies that STM retention in flies fed with $B$. monnieri had retained the learned behavior for a longer time compared to the group without B. monnieri supplementation.

Sleep-deprived flies fed with B. monnieri, when compared to those that are not fed with the supplement, retained the behavior longer indicating that the supplement compensates for the deficiency caused by sleep deprivation. The STM retention rates of the two highest concentrations were higher compared to that of the lowest concentration $(p<0.001)$. Looking at the mid and high concentrations, it appears that an increase in concentrations of $B$. monnieri supplementation did not translate to a corresponding increase in STM retention. The increase in STM retention of slp + and slp- flies, in the mid and high concentrations of $B$. monnieri supplement, reached similar levels as indicated by their APR values not being significantly different $(p>0.05)$. There was a decrease in the APR values of the group $\mathrm{slp}^{-} / \mathrm{Bm}^{+}$low in comparison to its non-sleep-deprived counterpart. Interaction between sleep deprivation and $B$. monnieri supplementation is not significant $(p>0.05)$ in the obtained STM retention rates.

Enhancement of STM retention of $\operatorname{slp}^{+} / \mathrm{Bm}^{+}$and $\mathrm{slp}^{-} /$ $\mathrm{Bm}^{+}$treatment groups also followed a dose-dependent manner. Compared to the $\mathrm{slp}^{+} / \mathrm{Bm}^{+}$group, a significant increase in STM retention in the $\mathrm{slp}^{-} / \mathrm{Bm}^{+}$was only observed in the two highest concentrations. This result suggests that if little experiential information was formed in the first place during the learning phase of the sleep-deprived flies, then the recently learned behavior that can be retained and retrieved for use in future encounters the effect of $B$. monnieri supplement on the differential and increasing STM retention trends which were likely to be manifestations of (1) enhancement of B. monnieri supplement on memory phase following a dose-dependent manner and (2) the natural events outlining the extinction of the memory of the conditioned behavior.

Although we did not study the underlying mechanism, previous studies of $B$. monnieri have identified its effects on neural transmission. Saini et al. (2010) evaluated the neuroprotective effects of B. monnieri extract on colchicine-induced cognitive impaired rat models, where the altered enzyme activities of $\mathrm{Na}^{+} /$ $\mathrm{K}^{+}$-ATPase was reported to be restored by B. monnieri. A study done by McPhee et al. (2016) showed that the administration of $B$. monnieri reduces neurological deficits together with increased $\mathrm{Na}^{+} /$ $\mathrm{K}^{+}$-ATPase activity. In this study, the release of intracellular $\mathrm{Ca}^{2+}$ was glutamate and neurotrophin-dependent, in the postsynaptic cell through a number of downstream protein kinases that are crucial for synaptogenesis. They observed a significant increase in cAMP response element-binding protein activity in the hippocampus after B. monnieri consumption. A study done by Hosamani and Muralidhara (2009) found that B. monnieri inhibited dopamine depletion, which are found to be altered during sleep deprivation. Furthermore, B. monnieri prevents apoptosis in neurons from oxidative stress (Sukumaran, 2019). These studies together suggest that $B$. monnieri protects neurons from oxidative stress which is induced by sleep deprivation.

\section{CONCLUSION}

We found that B. monnieri treatment ameliorated learning and STM loss induced by sleep deprivation in
Drosophila model. Supplementing fly food with B. monnieri not only enhanced the learning and STM retention deficits caused by sleep deprivation but also increased the learning and STM retention of these sleep-deprived flies to the same level of increase observed in non-sleep-deprived flies. The findings in this study might be applied to anticipated short-term sleep disturbances, such as temporary night shifts, staying up late for an exam, or jet lags. The inclusion of natural nootropic in a daily-based diet in humans may improve mental performance, without the risks of side effects. In dietary science, a proper combination of natural nootropics, known as nootropic stacking, synergistically enhances the efficacy of each active ingredient of the nootropic. In conclusion, this is an innovative study that may provide a new pharmacological treatment to aid in protecting learning deficits and memory loss in individuals with short-term sleep loss.

\section{CONFLICT OF INTEREST}

All the authors declare that they have no conflicts of interest for this work.

\section{FUNDING}

None.

\section{REFERENCES}

Abdul Manap AS, Vijayabalan S, Madhavan P, Chia YY, Arya A, Wong EH, Rizwan F, Bindal U, Koshy S. Bacopa monnieri, a neuroprotective lead in Alzheimer disease: a review on its properties, mechanisms of action, and preclinical and clinical studies. Drug Target Insights, 2019; 13:1177392819866412.

Bai L, Sehgal A. Anaplastic lymphoma kinase acts in the Drosophila mushroom body to negatively regulate sleep. PLoS Genet, 2015; 11(11).

Belenky G, Wesensten NJ, Thorne DR, Thomas ML, Sing HC, Redmond DP, Russo MB, Balkin TJ. Patterns of performance degradation and restoration during sleep restriction and subsequent recovery: a sleep dose-response study. J Sleep Res, 2003; 12(1):1-12.

Bhattacharya SK, Bhattacharya A, Kumar A, Ghosal S. Antioxidant activity of Bacopa monniera in rat frontal cortex, striatum and hippocampus. Phytother Res, 2000; 14(3):174-9.

Bhattacharya SK, Ghosal S. Anxiolytic activity of a standardized extract of Bacopa monniera: an experimental study. Phytomedicine, 1998; $5(2): 77-82$.

Brondino N, De Silvestri A, Re S, Lanati N, Thiemann P, Verna A, Emanuele E, Politi P. A systematic review and meta-analysis of Ginkgo biloba in neuropsychiatric disorders: from ancient tradition to modern-day medicine. Evid Based Complement Alternat Med, 2013; 2013:915691.

Chaudhari KS, Tiwari NR, Tiwari RR, Sharma RS. Neurocognitive effect of nootropic drug brahmi (Bacopa monnieri) in Alzheimer's disease. Ann Neurosci, 2017; 24(2):111-122.

Dag U, Lei Z, Le JQ, Wong A, Bushey D, Keleman K. Neuronal reactivation during post-learning sleep consolidates long-term memory in Drosophila. Elife, 2019; 8:e42786.

Deepak M, Amit A. The need for establishing identities of 'bacoside A and B', the putative major bioactive saponins of Indian medicinal plant Bacopa monnieri. Phytomedicine, 2004; 11(2-3):264-8.

Dissel, S. Drosophila as a model to study the relationship between sleep, plasticity, and memory. Front Physiol, 2020; 11:533.

Dubey T, Chinnathambi S. Brahmi (Bacopa monnieri): an ayurvedic herb against the Alzheimer's disease. Arch Biochem Biophys, 2019; 676:108153.

Duncko R, Cornwell B, Cui L, Merikangas KR, Grillon C. Acute exposure to stress improves performance in trace eyeblink conditioning and spatial learning tasks in healthy men. Learn Mem, 2007; 14(5):329-35. 
Foley BR, Marjoram P, Nuzhdin SV. Basic reversal-learning capacity in flies suggests rudiments of complex cognition. PLoS One, 2017; 12(8):e0181749.

Fukui K, Onodera K, Shinkai T, Suzuki S, Urano S. Impairment of learning and memory in rats caused by oxidative stress and aging, and changes in antioxidative defense systems. Ann N Y Acad Sci, 2001; 928:168-75.

Gregory AM, Sadeh A. Annual research review: sleep problems in childhood psychiatric disorders--a review of the latest science. J Child Psychol Psychiatry, 2015; 57(3):296-317.

Guarnieri DJ, Heberlein U. Drosophila melanogaster, a genetic model system for alcohol research. Int Rev Neurobiol, 2003; 54:199-228.

Havekes R, Abel T. The tired hippocampus: the molecular impact of sleep deprivation on hippocampal function. Curr Opin Neurobiol, 2017; 44:13-9.

Hosamani R, Muralidhara. Neuroprotective efficacy of Bacopa monnieri against rotenone induced oxidative stress and neurotoxicity in Drosophila melanogaster. Neurotoxicology, 2009; 30(6):977-85.

Huber R, Hill SL, Holladay C, Biesiadecki M, Tononi G, Cirelli C. Sleep homeostasis in Drosophila melanogaster. Sleep, 2004; 27(4):62839.

Klumpers UM, Veltman DJ, van Tol MJ, Kloet RW, Boellaard R, Lammertsma AA, Hoogendijk WJ. Neurophysiological effects of sleep deprivation in healthy adults, a pilot study. PLoS One, 2015; 10(1):e0116906.

LeVault KR, Tischkau SA, Brewer GJ. Circadian disruption reveals a correlation of an oxidative GSH/GSSG redox shift with learning and impaired memory in an Alzheimer's disease mouse model. J Alzheimers Dis, 2016; 49(2):301-16.

Li X, Yu F, Guo A. Sleep deprivation specifically impairs shortterm olfactory memory in Drosophila. Sleep. 2009; 32(11):1417-24.

Mahato SB, Garai S, Chakravarty AK. Bacopasaponins E and F: two jujubogenin bisdesmosides from Bacopa monniera. Phytochemistry, 2000; 53(6):711-4.

Margulies C, Tully T, Dubnau J. Deconstructing memory in Drosophila. Curr Biol, 2005; 15(17):R700-13.

McGuire SE, Deshazer M, Davis RL. Thirty years of olfactory learning and memory research in Drosophila melanogaster. Prog Neurobiol, 2005; 76(5):328-47.

McPhee GM, Downey LA, Noble A, Stough C. Cognitive training and Bacopa monnieri: evidence for a combined intervention to alleviate age associated cognitive decline. Med Hypotheses, 2016; 95:71-6.

Medina, PM, Cabaccan, JS, Asis, JL. Effect of natural and artificial sweeteners on the hemolymph glucose level (HGL) in Drosophila melanogaster. Int J Biosci, 2015; 7(1):119-31.

Nair AB, Jacob S. A simple practice guide for dose conversion between animals and human. J Basic Clin Pharmacol, 2016; 7:27-31.

Rauf K, Subhan F, Abbas M, ul Haq I, Ali G, Ayaz M. Effect of acute and sub chronic use of Bacopa monnieri on dopamine and serotonin turnover in mice whole brain. Afr J Pharm Pharmacol, 2012; 6:2767-74.
Riemensperger T, Völler T, Stock P, Buchner E, Fiala A. Punishment prediction by dopaminergic neurons in Drosophila. Curr Biol, 2005; 15(21):1953-60.

Saini N, Oelhafen S, Hua H, Georgiev O, Schaffner W, Büeler H. Extended lifespan of Drosophila parkin mutants through sequestration of redox-active metals and enhancement of anti-oxidative pathways. Neurobiol Dis, 2010; 40(1):82-92.

Seugnet L, Suzuki Y, Stidd R, Shaw PJ. Aversive phototaxic suppression: evaluation of a short-term memory assay in Drosophila melanogaster. Genes Brain Behav, 2009; 8(4):377-89.

Shaw PJ, Cirelli C, Greenspan RJ, Tononi G. Correlates of sleep and waking in Drosophila melanogaster. Science, 2000; 287:1834-7.

Singh HK, Dhawan BN. Effect of Bacopa monniera Linn. (brahmi) extract on avoidance responses in rat. J Ethnopharmacol, 1982; 5(2):205-14.

Singh A, Singh SK. Evaluation of antifertility potential of Brahmi in male mouse. Contraception, 2009; 79(1):71-9.

Sireeratawong S, Jaijoy K, Khonsung P, Lertprasertsuk N, Ingkaninan K. Acute and chronic toxicities of Bacopa monnieri extract in sprague-dawley rats. BMC Complement Altern Med, 2016; 16:249.

Sukumaran NP, Amalraj A, Gopi S. Neuropharmacological and cognitive effects of Bacopa monnieri (L.) Wettst - a review on its mechanistic aspects. Complement Ther Med, 2019; 44:68-82.

Tang S, Guo A. Choice behavior of Drosophila facing contradictory visual cues. Science, 2001; 294(5546):1543-7.

Thomas J, Overeem S, Dresler M, Kessels RPC, Claassen JAHR. Shift-work-related sleep disruption and the risk of decline in cognitive function: The CRUISE Study [published online ahead of print, 2020 Jun 8]. J Sleep Res, 2020; e13068.

Tsuda L, Lim YM. Alzheimer's disease model system using Drosophila. Adv Exp Med Biol, 2018; 1076:25-40.

Waser M, Lauritzen MJ, Fagerlund B, Osler M, Mortensen EL, Sørensen H, Jennum P. Sleep efficiency and neurophysiological patterns in middle-aged men are associated with cognitive change over their adult life course. J Sleep Res, 2019; 28:e12793.

Yang H, Xie J, Mu W, Ruan X, Zhang J, Yao L, Diao Z, Wu M, Li Y, Ren W, Han J. Tea polyphenols protect learning and memory in sleepdeprived mice by promoting AMPA receptor internalization. Neuroreport, 2020; 31(12):857-64.

How to cite this article:

Ong BAG, Villanueva MCN, Medina PMB. Bacopa monnieri supplementation increases learning and short-term memory retention of sleep-deprived Drosophila melanogaster. J Appl Pharm Sci, 2020; 10(12):104-110. 